\title{
Resolving 1 million sensing points in an optimized differential time-domain Brillouin sensor
}

\author{
Alejandro Dominguez-Lopez, ${ }^{1, *}$ Marcelo A. Soto, ${ }^{2}$ Sonia Martin-Lopez, ${ }^{1}$ \\ Luc Thevenaz, ${ }^{2}$ and Miguel Gonzalez-Herraez ${ }^{1}$ \\ ${ }^{1}$ Department of Electronics, University of Alcala, Polytechnic School, 28805 Alcala de Henares, Spain \\ ${ }^{2}$ EPFL, Swiss Federal Institute of Technology, Institute of Electrical Engineering, SCI STI LT, Station 11, CH-1015 Lausanne, Switzerland \\ *Corresponding author: alejandro.dominguez@@uah.es
}

Received 19 January 2017; revised 6 April 2017; accepted 6 April 2017; posted 7 April 2017 (Doc. ID 284561); published 3 May 2017

\begin{abstract}
A differential pulse-width pair (DPP) Brillouin distributed fiber sensor is implemented to achieve centimetric spatial resolution over distances of several kilometers. The presented scheme uses a scanning method in which the spectral separation between the two probe sidebands is kept constant, while the optical frequency of the pump is swept to scan the Brillouin spectral response. Experimental results show that this method avoids detrimental temporal distortions of the pump pulses, which in a standard implementation prevent the DPP method from operating over mid-to-long distances. Such a novel scanning procedure allows the resolving, for the first time in pure time-domain Brillouin sensors, of 1,000,000 sensing points, i.e., $1 \mathrm{~cm}$ spatial resolution over $10 \mathrm{~km}$ in a conventional acquisition time. (C) 2017 Optical Society of America
\end{abstract}

OCIS codes: (060.2370) Fiber optics sensors; (290.5900) Scattering, stimulated Brillouin; (060.4370) Nonlinear optics, fibers.

https://doi.org/10.1364/OL.42.001903

Brillouin-based distributed optical fiber sensors have become an established sensing technology due to their ability to measure strain/temperature changes along tens of kilometers of fiber, making them very suitable to monitor large civil infrastructures. In particular, in Brillouin optical time-domain analysis (BOTDA), the interaction of two counter-propagating signals, a pulsed pump, and a continuous-wave $(\mathrm{CW})$ probe signal allows for measuring the temperature- and straindependent Brillouin gain/loss response of the sensing fiber with a spatial resolution given by the pump pulse width [1]. The probe wave is most commonly generated by making use of double-sideband (DSB) intensity modulation to mitigate the depletion of the pump pulse power, particularly in long-range schemes [2]. However, it has been recently proven that DSB schemes also bring massive spectral and temporal distortion on the pump pulse at high probe powers, inducing severe deformations in the Brillouin gain and loss spectra, and ultimately impairing the Brillouin frequency shift (BFS) determination [3]. Such a distortion results from asymmetric gain and loss, affecting the pump pulse in the conventional probe sideband frequency scanning, and scales directly with the probe wave power. To operate in a safe range, i.e., within a power range that secures negligible spectral distortions, the probe power in such a BOTDA scheme is limited to roughly $-5 \mathrm{dBm}$ per sideband [3], thus limiting the best attainable signalto-noise ratio (SNR) and measurand precision [4].

On the other hand, the non-instantaneous $(\sim 10 \mathrm{~ns})$ response time of the acoustic wave generated at each fiber location imposes another limitation to the conventional BOTDA sensing technique: it impacts the spatial resolution, which cannot be reliably improved below $1 \mathrm{~m}$, using an isolated interrogating pulse. To overcome this limitation and achieve submetric spatial resolution, different approaches have been proposed based on frequency, correlation [5-9], or time[10-12] domain approaches. All of these techniques aim at increasing the number of resolved sensing points in the fiber. Frequency- and correlation-domain approaches allow very sharp spatial resolutions (in the order of millimeters or a few centimeters), however the measurement range is typically limited to a few kilometers. Using correlation-based schemes, a significant increase in the number of resolved points has been demonstrated along fibers of several kilometers-long [6-9]; however, the total measurement time still remains extremely long, e.g., a few hours for 1 million points [6]. A most recent improvement has ultimately led to a remarkable state-of-the-art record, doubling the number of resolved points up to 2.1 million, in spite of an eight-fold increase in the acquisition time [9].

Conversely, time-domain approaches are much faster and typically make use of differential pulses, such as differential pulsewidth pair (DPP) BOTDA $[10,11]$ or Brillouin echoes (BEDS) [12]. The spatial resolution in those cases highly depends on the rising/falling time of the used optical pulses, thus typically being limited to tens of centimeters along mid-range sensing distances (usually below $10 \mathrm{~km}$ ). In the literature, it can be found that a spatial resolution of $2 \mathrm{~cm}$ can be secured along the $2 \mathrm{~km}$ range using a DPP-BOTDA system [11], while a $5 \mathrm{~cm}$ spatial resolution has been demonstrated with BEDS along a $5 \mathrm{~km}$ range [12]. Extending the sensing range turns out to be very challenging; thus, a sensing range of $60 \mathrm{~km}$ has only been demonstrated with a $25 \mathrm{~cm}$ spatial resolution, making use of optical pulse coding and representing $250 \mathrm{k}$ of independent points [13]. 
The DPP technique $[10,11]$ is based on the subtraction of two temporal traces, each of them obtained using long pump pulse widths of slightly different durations. The resulting spatial resolution is then determined by the difference between the pulse widths. Typically, the long pulse's duration is chosen to be large enough to properly build up the acoustic wave (e.g., 50-60 ns). In such a case, however, the polarization noise (which is a fraction of the measured traces) becomes dominant in the differential trace. To secure correct DPP measurements, this polarization noise amplitude must be kept below the differential trace amplitude, which scales with the differential pulse width. Reducing the width of the long pulses implies less relative polarization noise in the differential trace, but also a shorter interaction length and, hence, a weaker response. In summary, there is an optimum to be found in the width of the pair of long pulses when aiming to reach very high spatial resolution.

In this Letter, we demonstrate that the limitations to extend the sensing range or to improve the spatial resolution of differential pulse techniques are not decisively due to the reduced measurement SNR, resulting from the larger attenuation in longer fibers, particularly critical considering the lower energy contained in shorter differential pulses, but are primarily due to spectral and temporal distortions affecting the pump pulses, and ultimately due to the limited polarization noise elimination. Mitigating these distortions secures high spatial resolution sensing along extended distances, as recently demonstrated in [14], where preliminary results have been reported with a $20 \mathrm{~cm}$ spatial resolution over $37.5 \mathrm{~km}$ of fiber. Here, we demonstrate that by using an optimized combination of a DPP-BOTDA scheme and a novel method for scanning the pump-probe frequency offset allows us to mitigate the detrimental pulse distortions and to reach a number of resolved points unmatched so far by time-domain schemes. In particular, we present here, for the first time to the best of our knowledge, a time-domain Brillouin sensor capable of resolving 1 million points, representing a significant improvement in the measurement time with respect to the state-of-the-art, since the full measurement time still remains of the order of a few minutes.

Aiming to avoid non-local effects occurring for strong probe powers, two effective solutions have been recently put forward in the literature $[3,15]$. In this case, in order to eliminate such pump pulse distortions and ensure sharp differential pulses for high spatial resolution sensing, we make here use of our previously proposed solution [3], in which the probe wave modulation frequency is kept fixed, matching the dominating Brillouin shift of the fiber. This way, a perfect overlap of the gain and loss processes generated by the anti-Stokes and Stokes components of the probe wave is secured, as shown in Fig. 1. This leads to a fully spectrally compensated gain and depletion over the pump, and consequently, inducing no distortion on it. The Brillouin gain or loss is then scanned by either sweeping the probe frequencies asymmetrically around the pump, or, alternatively, by scanning the pump pulse frequency as described in [3]. Note that the non-distorted behavior holds for all scanned frequencies, unlike the conventional probe frequency scanning scheme, in which no distortion is only secured when the modulation frequency matches the BFS of the fiber.

By combining DPP-BOTDA with the above-described scanning technique [3], the pulse distortion can be significantly mitigated, making it possible to increase the probe power launched into the fiber (theoretically limited by the onset of

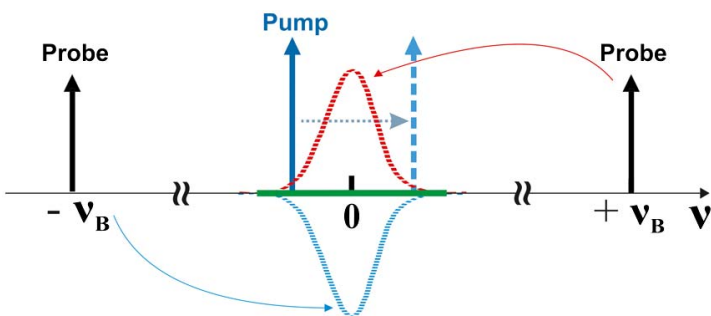

Fig. 1. Illustration of the proposed scanning technique, where the probe wave remains frequency-fixed at $\pm \nu_{B}$, inducing no distortion over the pump pulse. The pump frequency is swept to properly scan Brillouin gain and loss curves.

amplified spontaneous Brillouin scattering [3]), enhancing the SNR of the sensor, while securing very sharp spatial resolution. To demonstrate this, a DPP-BOTDA system has been implemented, as shown in Fig. 2, using the novel scanning technique described in Fig. 1. In this scheme, the probe sidebands remain frequency-fixed while the pump pulse scans the Brillouin gain or loss spectrum. First, the laser beam is DSB modulated by means of an intensity electro-optic modulator (EOM1), whose frequency is set to match the average BFS over the last section of the sensing fiber (equivalent to the non-linear effective length) [3]. The modulated CW is then split into distinct branches; one of them (the lower branch in Fig. 2) already constitutes the probe wave and is amplified by an erbiumdoped fiber amplifier (EDFA 1) in order to increase the power launched into the sensing fiber (and, hence, the SNR in detection). Right before being launched into the fiber-under-test (FUT), the probe goes through a polarization synthesizer (P. Synth), which is used to reduce polarization fading in the measured temporal traces.

The other branch (the upper one in Fig. 2) is used to generate the pump pulse. To do so, it is first necessary to select either the upper or lower modulation sidebands by means of a suitable filter. In our implementation, a narrow tunable filter ( $\sim 80 \mathrm{pm}$ bandwidth) is used to select the upper frequency sideband, which is then intensity-modulated by means of a second modulator (EOM2) at a frequency difference sweeping around the BFS $\left(\nu_{B} \pm \Delta \nu\right)$. One of the two new frequency components is selected using another tunable filter, whose bandwidth is wide enough to enable frequency tuning over the selected spectral span, but narrow enough to filter out all the unwanted spectral components. Subsequently, the pulse is properly shaped using an intensity modulator (EOM3), and then amplified by an EDFA (EDFA2).

To increase the extinction ratio (ER) of the pulses (30-40 dB at the EOM output), an electro-optical microelectromechanical system (MEMS) switch (ES) is used to gate the pulses and ensure an overall ER higher than $80 \mathrm{~dB}$. Before entering the circulator, the pulses go through a fast polarization scrambler, which helps by reducing the polarization fading in the traces (in addition to the P. Synth). After selecting the Brillouin loss component by means of a dense wavelength division multiplexing (DWDM) filter, the signal is fed into a high-bandwidth $(9.5 \mathrm{GHz})$ photo-receiver.

In order to analyze the performance of the above-explained technique, full DPP-BOTDA measurements have been carried out over a $10 \mathrm{~km}$ single mode fiber (SMF), presenting a homogeneous $\mathrm{BFS}$ of $\sim 10.855 \mathrm{GHz}$ along the entire fiber. 


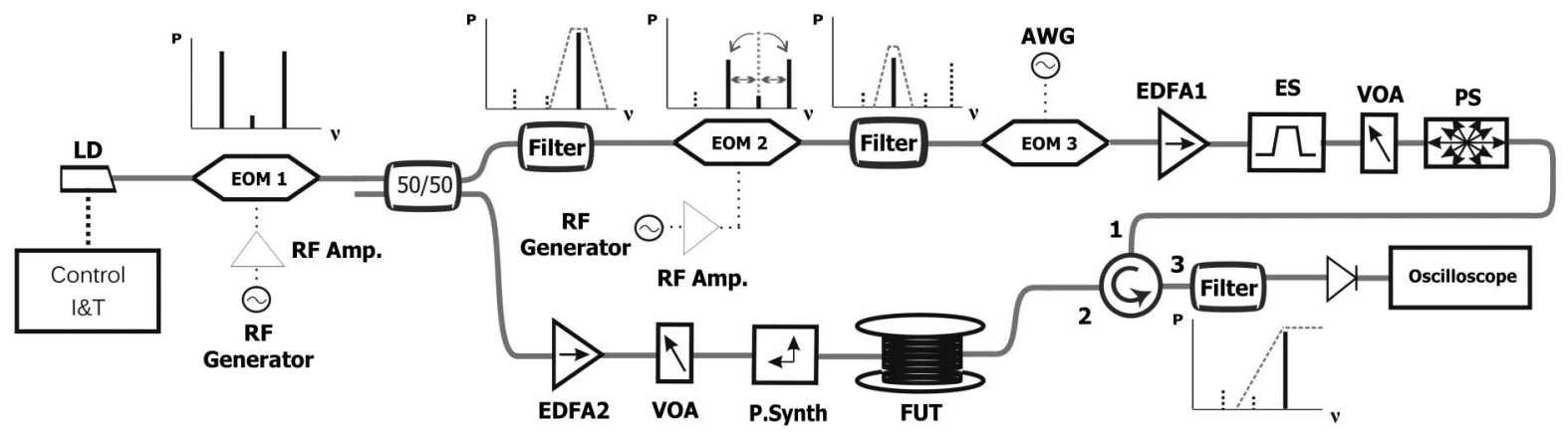

Fig. 2. Experimental setup. LD, laser diode; EOM, electro-optical modulator; AWG, arbitrary waveform generator; EDFA, erbium-doped fiber amplifier; VOA, variable optical attenuator; P.Synth, polarization synthesizer; ES, electrical switch; PS, polarization switch; FUT, fiber-under-test.

In the measurements, sharp squared pulses (100 ps fall time) of 26.4 and 26.3 ns have been used, thus, resulting in a spatial resolution of $1 \mathrm{~cm}$ (100 ps width difference). Such pulse widths are short enough as to mitigate polarization fluctuations in the BOTDA traces and wide enough to generate a sufficient Brillouin interaction to enable the DPP technique. The pump pulse peak power is $\sim 100 \mathrm{~mW}$, while the probe wave is $\sim 2 \mathrm{~mW}$ per sideband. To evaluate the impact of the proposed scanning method on the reduction of the pulse distortion, pulses are measured at the output of the sensing fiber, i.e., after propagating along the $10-\mathrm{km}$-long fiber and interacting with the probe wave through stimulated Brillouin scattering (SBS). Figure 3(a) shows the acquired long pump pulses and the resulting differential pulse (subtraction among long pulses), using the conventional scanning method. Measured shapes illustrate the temporal distortion experienced by the two long pump pulses when the pump-probe frequency offset is detuned by $+25 \mathrm{MHz}$ from the average BFS. It can be observed that such a non-negligible temporal distortion leads to a complete jamming of the differential pulse, resulting from the subtraction of the long and short pulses [red line in Fig. 3(a)], thus making the DPP technique not reliable for this sensing range and probe powers. However, as it can be seen in Fig. 3(b), by making use of the proposed scanning procedure, the long pump pulses maintain their original shape along the entire fiber, regardless of the probe power and frequency detuning. This behavior secures the correct operation of the DPPBOTDA technique, showing a well-shaped differential pulse of 100 ps along the entire sensing fiber. It should, however, be mentioned that a penalty $(\sim 2 \mathrm{~dB})$ on the peak power of the differential pulse can be observed, which results from the limited bandwidth of the pulse generator used in the experiment (defining a pulse falling time of 100 ps, being similar to the expected differential pulse width) and from the expected non-maximum differential gain given for the selected pair of pulses (see Fig. 1 in Ref. [13] for a similar study).

Note that in the implemented DPP-BOTDA sensing scheme, the intended spatial resolution $(1 \mathrm{~cm})$ is obtained by subtracting traces measured with pump pulses of 26.4 and $26.3 \mathrm{~ns}$, which means that the 100 ps differential pulse corresponds to $\sim 0.3 \%$ of the total pulse width. In such a scenario, minimizing polarization fading in the trace and noise resulting from time-varying polarization issues turns out to be essential. Thus, a redundant polarization scrambling scheme has been utilized in both the pump and probe waves. On one
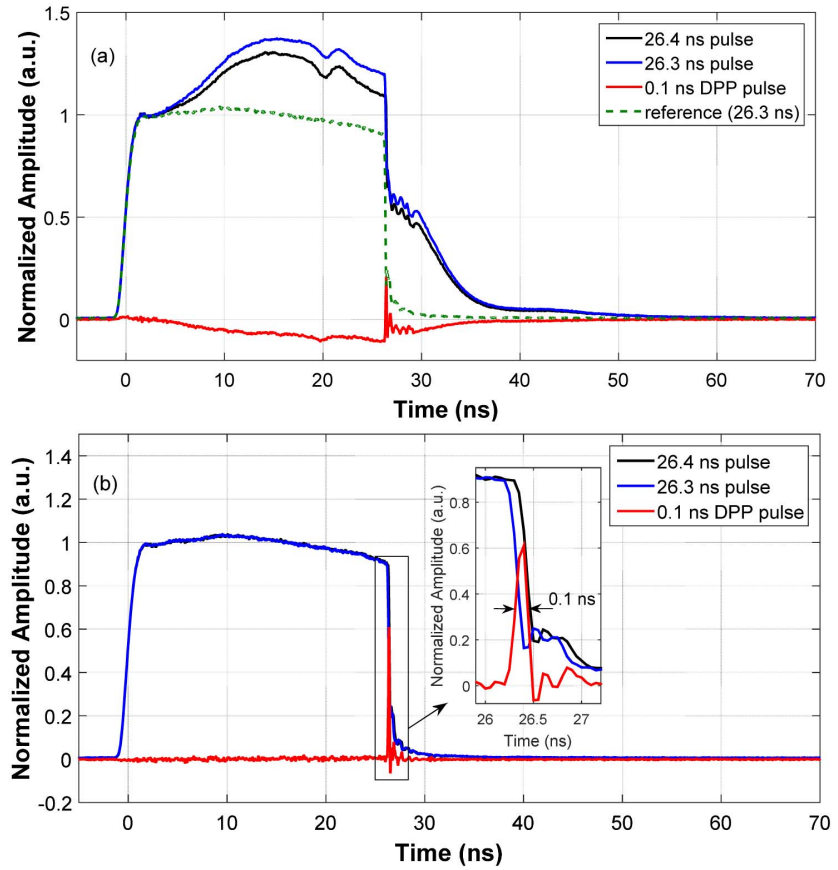

Fig. 3. DPP working principle, presenting the long and short pulses (black and blue, respectively), and the resulting differential pulse (red). (a) DPP on the conventional BOTDA sweeping method, showing a distortion in the pulses for a $+25 \mathrm{MHz}$ pump-probe frequency detuning (non-distorted $26.3 \mathrm{~ns}$ pulse is shown in green dashed lines for reference) and a null differential pulse. (b) DPP-BOTDA using the proposed scanning procedure: both long pulses remain well-shaped, while the differential pulse shows a correct shape and the expected 100 ps width.

side, the pump is scrambled by means of a fast polarization scrambler. On the other side, the probe goes through a P. Synth, which sets its state to six different polarization states (three pairs of orthogonal polarization states). This way, the impact of polarization fading and time-varying polarization issues on the differential time-domain traces can be substantially reduced, thus providing a reliable differential trace measurement, as shown in Fig. 4(a). Although the differential trace shown in Fig. 4(a) looks to have large amplitude fluctuations, the SNR calculated at the end of the sensing fiber (around $10 \mathrm{~km}$ distance) is $\sim 4.2 \mathrm{~dB}$, thus securing a large enough signal contrast for reliable BFS determination [4]. 

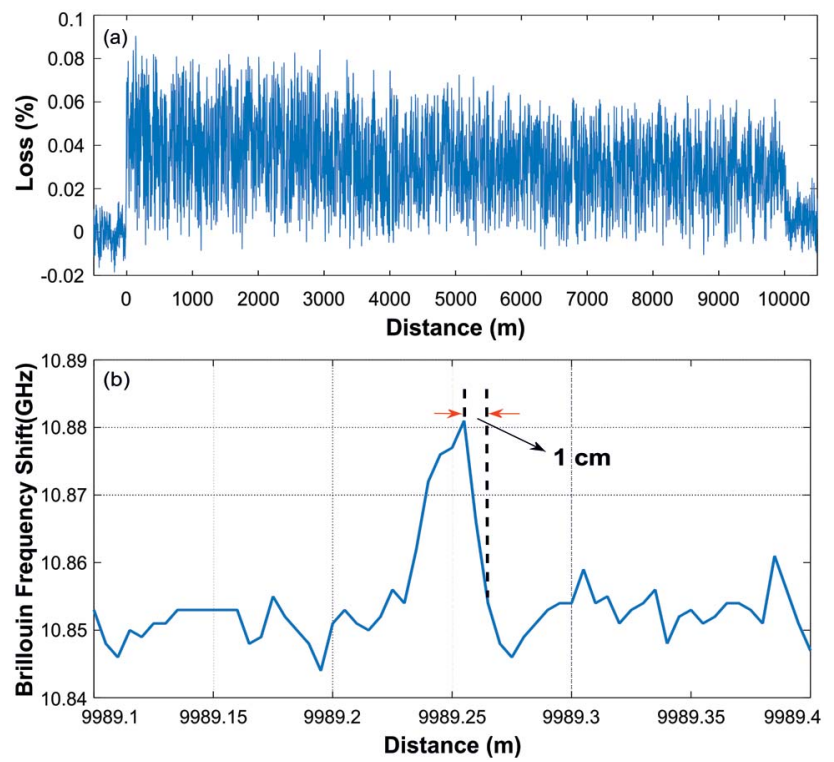

Fig. 4. (a) DPP-BOTDA trace along the $10 \mathrm{~km}$ fiber using a differential pulse of $100 \mathrm{ps}(1 \mathrm{~cm}$ resolution) at the peak frequency of $10.855 \mathrm{GHz}$; (b) BFS evolution at the far end of the fiber showing a $3 \mathrm{~cm}$ hot-spot presenting a BFS offset by $-30 \mathrm{MHz}$ with respect to the unheated fiber.

The system performance has been finally evaluated by creating a $3 \mathrm{~cm}$ hot-spot at the end of the FUT by attaching a resistor to the fiber section, and applying a current high enough to heat the fiber up to $\sim 56^{\circ} \mathrm{C}$. A full frequency sweep has been carried out, where each temporal trace has been averaged 18,000 times. The evolution of the BFS along the FUT has been obtained by fitting a parabolic curve to the measured Brillouin loss spectrum [4], and a zoom-in of the last few meters of fiber is presented in Fig. 4(b). The measured BFS evolution demonstrates the presence of a hot-spot around $9989 \mathrm{~m}$. The heated section shows a BFS at $\sim 10.880 \mathrm{GHz}$ offset by $\sim 30 \mathrm{MHz}$ when compared to the rest of the fiber at room temperature $\left(\sim 25^{\circ} \mathrm{C}\right)$, thus confirming the $\sim 30^{\circ} \mathrm{C}$ increase when using the estimated conversion factor of $1 \mathrm{MHz} /{ }^{\circ} \mathrm{C}$. The measured hot-spot width is $3 \mathrm{~cm}$ with a transient distance of $1 \mathrm{~cm}$, demonstrating the expected spatial resolution of the DPP-BOTDA sensor.

The frequency uncertainty measured in this case at the end of the fiber is $\sim 3.1 \mathrm{MHz}$, being in agreement with the theoretical expected value based on the measurement SNR [4]. It should be highlighted that the achieved spatial resolution of $1 \mathrm{~cm}$ over a sensing range of $10 \mathrm{~km}$ represents an equivalent of 1,000,000 resolved points, which constitutes a record number of points using a Brillouin time-domain sensor. Compared to correlationdomain methods reaching the same number of points, the DPP-BOTDA scheme implemented here leads to a significant reduction in the measurement time, from several hours down to only a few minutes. In particular, in our experiment, the measurement time has been reduced down to $\sim 20 \mathrm{~min}$.

It should be further noted that the use of the proposed scanning method in DPP-BOTDA turns essential when trying to achieve such extreme performance, where the conventional sweeping procedure has proven to fail. To secure a comparable SNR using the conventional scanning method, i.e., avoiding non-local effects on the pulses, the probe power would need to be reduced by roughly one order of magnitude compared to the one used in the current method. Thus, to obtain a comparable performance, it would require a vast increase in the amount of averages by roughly 2 orders of magnitude, and consequently, significantly expanding the total acquisition time.

In conclusion, fundamental limitations imposed by temporal distortions of pump pulses in a DPP-BOTDA sensor, and up to now preventing mid-to-long-range high spatial resolution sensing, have been mitigated here using a novel method for frequency scanning. The proposed technique ensures nondistorted pump pulses (time and frequency wise). while enabling it to raise the probe wave power launched into the sensing fiber (ultimately limited by the onset of amplified spontaneous Brillouin scattering). Such a power increase enhances the overall system performance, enabling it in this case to improve the spatial resolution down to $1 \mathrm{~cm}$ over an extended sensing range of $10 \mathrm{~km}$. This result is, to the best of our knowledge, the first demonstration of a time-domain Brillouin sensor capable of resolving 1,000,000 sensing points along a single optical fiber. Given the time-domain nature of the implemented scheme, the measurement time can be substantially reduced from the hour scale (typically required by correlation-domain Brillouin sensing to scan 1 million points) down to a few minutes. In terms of figure-of-merit [4], the present method yields a seven-fold improvement when compared to other time-domain approaches [11]. The potential improvement provided by the present approach is very high, as it could also be combined with other additional techniques for performance enhancement, such as Raman amplification, pulse coding, and/or image processing.

Funding. H2020 European Research Council (ERC) (307441); FINESSE (MSCA-ITN-ETN-722509); DOMINO Water JPI project; Ministerio de Economía y Competitividad (MINECO) (TEC2013-45265-R, TEC2015-71127-C2-2-R); Ramón y Cajal (SINFOTON-CM-S2013/MIT-2790).

Acknowledgment. The work of S. M. L. was supported by MINECO through a "Ramón y Cajal" Contract.

\section{REFERENCES}

1. T. Horiguchi, K. Shimizu, T. Kurashima, M. Tateda, and Y. Koyamada, J. Lightwave Technol. 13, 1296 (1995).

2. L. Thévenaz, S. F. Mafang, and J. Lin, Opt. Express 21, 14017 (2013).

3. A. Dominguez-Lopez, Z. Yang, M. A. Soto, X. Angulo-Vinuesa, S. Martin-Lopez, L. Thevenaz, and M. Gonzalez-Herraez, Opt. Express 24, 10188 (2016).

4. M. A. Soto and L. Thévenaz, Opt. Express 21, 31347 (2013).

5. K. Hotate and T. Hasegawa, IEICE Trans. Electron. E83-C, 405 (2000).

6. A. Denisov, M. A. Soto, and L. Thevenaz, Proc. SPIE 9157, 9157D2 (2014).

7. Y. London, Y. Antman, E. Preter, N. Levanon, and A. Zadok, J. Lightwave Technol. 34, 4421 (2016).

8. Y. H. Kim, K. Lee, and K. Y. Song, Opt. Express 23, 33241 (2015).

9. A. Denisov, M. A. Soto, and L. Thevenaz, LightSci. Appl.5, e16074 (2016)

10. W. Li, X. Bao, Y. Li, and L. Chen, Opt. Express 16, 21616 (2008).

11. Y. Dong, H. Zhang, L. Chen, and X. Bao, Appl. Opt. 51, 1229 (2012).

12. S. M. Foaleng, M. Tur, J.-C. Beugnot, and L. Thévenaz, J. Lightwave Technol. 28, 2993 (2010).

13. M. A. Soto, M. Taki, G. Bolognini, and F. Di Pasquale, Opt. Express 20, 6860 (2012)

14. A. Dominguez-Lopez, M. A. Soto, S. Martin-Lopez, M. GonzalezHerraez, and L. Thevenaz, Proc. SPIE 9916, 991635 (2016).

15. R. Ruiz-Lombera, J. Urricelqui, M. Sagues, J. Mirapeix, J. M. López-Higuera, and A. Loayssa, IEEE Photon. J. 7, 1 (2015). 